University of Nebraska - Lincoln

DigitalCommons@University of Nebraska - Lincoln

June 2001

\title{
Layer-resolved spin polarization in Sb overlayers on NiMnSb
}

\author{
Ralph Skomski \\ University of Nebraska-Lincoln, rskomski2@unl.edu \\ T. Komesu \\ University of Nebraska-Lincoln, tkomesu2@unl.edu
}

C.N. Borca

University of Nebraska-Lincoln

H.-K. Jeong

University of Nebraska-Lincoln, hjeong@unl.edu

Peter A. Dowben

University of Nebraska-Lincoln, pdowben@unl.edu

See next page for additional authors

Follow this and additional works at: https://digitalcommons.unl.edu/physicsdowben

Part of the Physics Commons

Skomski, Ralph; Komesu, T.; Borca, C.N.; Jeong, H.-K.; Dowben, Peter A.; Ristoiu, D. ; and Nozie'res, J.P., "Layer-resolved spin polarization in Sb overlayers on NiMnSb" (2001). Peter Dowben Publications. 24. https://digitalcommons.unl.edu/physicsdowben/24

This Article is brought to you for free and open access by the Research Papers in Physics and Astronomy at DigitalCommons@University of Nebraska - Lincoln. It has been accepted for inclusion in Peter Dowben Publications by an authorized administrator of DigitalCommons@University of Nebraska - Lincoln. 


\section{Authors}

Ralph Skomski, T. Komesu, C.N. Borca, H.-K. Jeong, Peter A. Dowben, D. Ristoiu, and J.P. Nozie'res 


\title{
Layer-resolved spin polarization in Sb overlayers on NiMnSb
}

\author{
R. Skomski, $\left.{ }^{a}\right)$ T. Komesu, C. N. Borca, H.-K. Jeong, \\ and P. A. Dowben \\ Department of Physics and Astronomy and Center for Materials Research and Analysis, \\ University of Nebraska, Lincoln, Nebraska 68588 \\ D. Ristoiu and J. P. Nozières \\ CNRS, Laboratoire Louis Néel, Avenue des Martyrs BP 166, 38042 Grenoble, CEDEX 09, France
}

The magnetism of antimony overlayers on a ferromagnetic substrate is investigated by spin-polarized inverse photoemission and explained in terms of a spin-dependent envelope-function approximation (SDEFA). The atomic structure of the films, which were deposited by sputtering $\mathrm{Sb}$ onto a $\mathrm{NiMnSb}(001)$ substrate, is characterized by a unique combination of three features: (i) $\mathrm{NiMnSb}$ is a highly spin-polarized semi-Heusler alloy predicted to be halfmetallic, (ii) antimony is a semimetal, exhibiting a band structure reminiscent of indirect-gap semiconductors, and (iii) the small lattice mismatch ensures a well-controlled interface. Combined x-ray absorption spectroscopy and spin-polarized inverse photoemission yield a layer-resolved spin polarization decaying on a length scale of the order of $1 \mathrm{~nm}$. The unusual range of the spin polarization in the paramagnetic overlayer is explained by considering the alloy-antimony interface as a spin-dependent perturbation potential and taking into account the low effective masses of the Sb conduction electrons (only about 0.1 for both electrons and holes). (C) 2001 American Institute of Physics.

[DOI: $10.1063 / 1.1357849]$

\section{INTRODUCTION}

Interfaces are well known to have a profound effect on the magnetic properties of the adjacent regions. Of particular interest are interfaces between different classes of materials, such as interfaces between magnetically ordered and semiconducting materials. ${ }^{1-3}$ This work focuses on the magnetism of heterostructures consisting of halfmetals and semimetals (Fig. 1). Halfmetallic materials, such as the ferromagnetic oxide $\mathrm{CrO}_{2}$ and the semi-Heusler alloy $\mathrm{NiMnSb}$, are ferromagnets characterized by the absence of minority $(\downarrow)$ electrons at the Fermi level-the minority band is half-filled whereas the majority $(\uparrow)$ electrons yield metallic conductivity. In other words, halfmetals are characterized by the coexistence of insulating behavior for minority electrons and metallic behavior for majority electrons. Semimetals, such as $\mathrm{C}$ and $\mathrm{Sb}$, are reminiscent of ordinary paramagnetic semiconductors, except that their "energy gap" is negative.

This work deals with NiMnSb layers covered by $\mathrm{Sb}$ overlayers. NiMnSb is a halfmetallic semi-Heusler alloy crystallizing in the cubic $\mathrm{Cl}_{b}$ structure. Ground-state theory predicts that $\mathrm{NiMnSb}$, a derivate of the parent Heusler alloy $\mathrm{Ni}_{2} \mathrm{MnSb}$, has a minority band gap of less than about 0.5 $\mathrm{eV} .{ }^{4,5}$ Antimony is a semimetal characterized by a very small negative energy gap; ${ }^{6,7}$ the overlap and Fermi energies are about 180 and $90 \mathrm{meV}$, respectively, and the electron and

${ }^{\text {a)} E l e c t r o n i c ~ m a i l: ~ r s k o m s k i @ u n l s e r v e . u n l . e d u ~}$ hole carrier densities are of comparable magnitude (about 5 $\left.\times 10^{-19} \mathrm{~cm}^{-3}\right){ }^{7}$ The band structure of the two materials is illustrated in Fig. 2. The preparation and characterization of the sputtered NiMnSb films considered in this work has been describedelsewhere. ${ }^{8,9}$ The films grown on $\mathrm{MgO}(001) / \mathrm{Mo}$ are epitaxial with a $5.9 \AA$ lattice constant and an (001) orientation. The $\mathrm{Sb}$ grows epitaxially on NiMnSb, with a $\langle 100\rangle$ orientation, a cubic structure, and a $3.1 \AA$ lattice constant. ${ }^{10}$

\section{Sb SPIN POLARIZATION}

The magnetism of the films was investigated by spinpolarized inverse photoemission. ${ }^{11}$ Figure 3 shows the spin

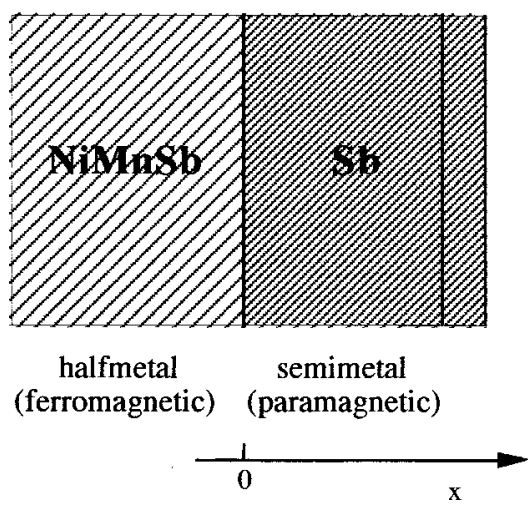

FIG. 1. Film cross section (schematic). Only the Sb surface layer is probed by inverse photoemission experiment. 

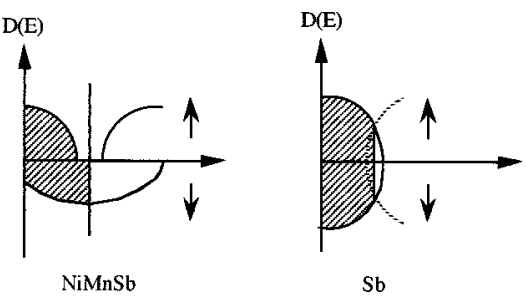

FIG. 2. Schematic densities of states of NiMnSb and Sb.

asymmetry at $E_{F}$ as a function of the Sb layer thickness. Only the $\mathrm{Sb}$ top layer is probed by this method (denoted by the arrow in Fig. 1), and the measured spin asymmetry characterizes, in crude way, the magnetic polarization of that layer. The lengths in Fig. 3 are given in arbitrary units, because it was not possible to obtain an exact value for the total coverlayer thickness, but in any case the thickness range shown in Fig. 3 is of the order of $1 \mathrm{~nm}$.

To explain the unusual range of the spin polarization in the $\mathrm{Sb}$ overlayer we take into account that there are no minority states available at the NiMnSb Fermi level. Figure 4 illustrates the basic idea of this spin-dependent envelope function approximation (SDEFA): the majority electrons are able to move from the $\mathrm{Sb}$ overlayer into the $\mathrm{NiMnSb}$, whereas the minority electrons are reflected at the $\mathrm{NiMnSb/Sb}$ interface. Let us make the crude model assumptions that the height of the reflecting barrier is much higher than the Fermi level and that the motion of the majority electrons is essentially free. Since the wave functions of free electrons are of the type $\exp (i \mathbf{k} \cdot \mathbf{r})$, the majority electron density remains unchanged in the $\mathrm{NiMnSb} / \mathrm{Sb}$ interface region. By contrast, the rigid-wall boundary condition for the minority electrons means that the wave functions $\psi_{\mathbf{k} \downarrow}(\mathbf{r})$ are equal to zero at $x=0$. This phenomenon is equivalent to the envelope function approximation used to describe quantum confinement in semiconductors, ${ }^{12}$ except that we consider spindependent wave function and a semi-infinite medium rather than a thin film. Using the solution of the spin-independent scattering of a three-dimensional free electron gas at an infinite potential barrier ${ }^{13}$ we obtain the electron density

$$
n_{\downarrow}(x)=\frac{k_{F} 3}{3 \pi^{2}}\left(1+3 \frac{\cos \left(2 k_{F} x\right)}{\left(2 k_{F} x\right)^{2}}-3 \frac{\sin \left(2 k_{F} x\right)}{\left(2 k_{F} x\right)^{3}}\right) .
$$

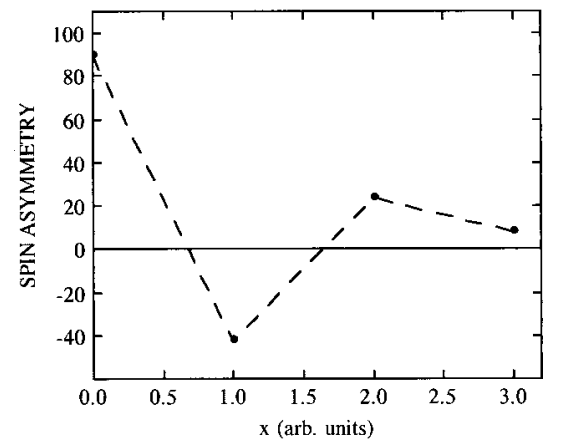

FIG. 3. Spin-polarized inverse photoemission data at $E_{F}$. The spin asymmetry is measured in percent.

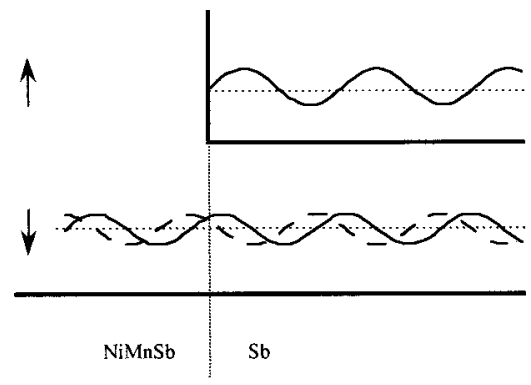

FIG. 4. Reflection of majority and minority electrons at the $\mathrm{NiMnSb} / \mathrm{Sb}$ interface.

The corresponding spin polarization of the carrier electrons, $m=\left(n_{\uparrow}-n_{\downarrow}\right) /\left(n_{\uparrow}+n_{\downarrow}\right)$, where $n_{\uparrow}=3 \pi^{2} / k_{F^{3}}$, is given by

$$
m(x)=\frac{k_{F^{3}}-3 \pi^{2} n(x)}{k_{F^{3}}+3 \pi^{2} n(x)} .
$$

The function $m_{x}(z)$ is shown in Fig. 5. Comparison of Figs. 3 and 5 shows that the present theory gives a qualitatively correct interpretation of the inverse photoemission data. The main point is that the small $\mathrm{Sb}$ carrier concentrations, the small effective mass $m^{*}$, and the associated low Fermi level lead to very small values of $k_{F}$.

\section{DISCUSSION}

Equations (1) and (2) establish a type of $R K K Y$-type theory for the spin polarization. As in the original $R K K Y$ theory,${ }^{14}$ the spin inhomogeneity originates from the fact that the electrons' finite wave vectors make it impossible to match perturbations on a local scale. Since we consider an infinite potential barrier, the present theory is actually nonperturbative, in contrast to the perturbative $R K K Y$ theory. However, the main difference between the $R K K Y$ theory and our approach is the semi-infinite character of the our problem. This relationship must be compared with a recent approach by Hunziker and Landolt, ${ }^{3}$ where spin effects in semiconductors are discussed by considering the Heisenberg exchange between two hydrogen-like orbitals characterized by a small effective mass $m^{*}$. (In this respect, $\mathrm{Sb}$ behaves like semiconductor barriers for majority and minority. Note that the use of Heisenberg-type exchange integrals is a fair

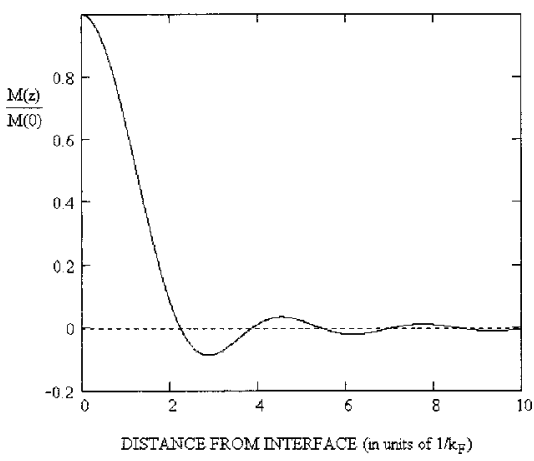

FIG. 5. The theoretical spin polarization in the $\mathrm{Sb}$ overlayer as a function of the distance from the $\mathrm{NiMnSb} / \mathrm{Sb}$ interface. 
alternative to the $R K K Y$ exchange, whereas the use hydrogen-like orbital amounts to a very crude treatment of the film geometry.

In a sense, the present theory describes the nonballistic injection of majority electrons from $\mathrm{NiMnSb}$ into $\mathrm{Sb}$, and the electron density implied by Fig. 4 is an equilibrium distribution, as in an ordinary diffusive semiconductor. Continuously injected electrons keep their spin orientation for some time, but they also create a self-consistent spin-dependent exchange field in the Sb overlayer, which establishes the observed equilibrium magnetization.

Due to the crude approximations involved, it is not possible to extract reliable quantitative information from Eqs. (1) and (2). For example, the assumptions of zero and infinite potential barriers for majority and minority electrons, respectively, are very crude, although the reduction of the minorityspin barrier from infinity to the band-gap energy does not alter the key predictions of this theory. Another issue is that valence and conduction bands cannot be considered as free electrons because they are far away from the $\Gamma$ point. ${ }^{7}$ This gives rise to short-range oscillations not considered in this work and means that the long-range magnetic response is realized by a small range of wave vectors $\Delta \mathbf{k}=\mathbf{k}-\mathbf{k}_{0} \ll \mathbf{k}_{0}$.

An interesting problem is the interpretation of the spin polarization in terms of a Ginzburg-Landau theory. In a linearized version, the theory has the form ${ }^{15}$

$$
C \frac{d^{2} m}{d x^{2}}+A m=h
$$

where $C$ and $A$ are phenomenological constants incorporating both quantum-mechanical ${ }^{14}$ and thermodynamical ${ }^{16}$ features. This equation approximates $m(x)$ as an exponentially decaying function, and from Eqs. (1) and (2) we deduce that $A / C$ scales as $1 / k_{F}$. Due to the smallness of $E_{F}$, finitetemperature effects are not necessarily negligible. A key effect is that thermal excitations tend to reduce the decay length by involving excited electrons with very short wavelengths. (This aspect of the problem will be discussed elsewhere).

\section{CONCLUSIONS}

In this work, we have investigated the problem of (equilibrium) spin injection from the half-metal NiMnSb into the semimetal $\mathrm{Sb}$. The present approach yields a layer-specific analysis of the spin polarization, in contrast to methods such as that used in Ref. 3, where spin injection is probed indirectly, by considering the exchange coupling through a semiconducting medium. The comparatively long range of the $\mathrm{Sb}$ spin polarization is explained by the semimetallic character of the $\mathrm{Sb}$ overlayer.

\section{ACKNOWLEDGMENT}

This work was supported by DOE, ONR, and CMRA.

${ }^{1}$ P. Walser, M. Schleberger, P. Fuchs, and M. Landolt, Phys. Rev. Lett. 80, 2217 (1998).

${ }^{2}$ Zh.-P. Shi and B. M. Klein, J. Appl. Phys. 79, 4776 (1996).

${ }^{3}$ M. Hunziker and M. Landolt, Phys. Rev. Lett. 84, 4713 (2000).

${ }^{4}$ J. G. Booth, Ferromagnetic Transition Metal Intermetallic Compounds, in Ferromagnetic Materials, Vol. 4, edited by E. P. Wohlfarth and K. H. J. Buschow (Elsevier, Amsterdam, 1988), pp. 211-308.

${ }^{5}$ R. A. de Groot and K. H. J. Buschow, J. Magn. Magn. Mater. 54-57, 1377 (1986)

${ }^{6}$ X. Gonze, J.-P. Michenaud, and J.-P. Vigneron, Phys. Rev. B 41, 11827 (1990).

${ }^{7}$ Y. Liu and R. E. Allen, Phys. Rev. B 52, 1566 (1995).

${ }^{8}$ C. N. Borca, T. Komesu, H.-K. Jeong, P. A. Dowben, D. Ristoiu, Ch. Hordequin, J. Pierre, and J. P. Nozieres, Appl. Phys. Lett. 77, 88 (2000).

${ }^{9}$ D. Ristoiu, J. P. Nozieres, C. N. Borca, B. Borca, and P. A. Dowben, Appl. Phys. Lett. 76, 2349 (2000).

${ }^{10}$ D. Ristoiu, J. P. Nozieres, C. N. Borca, T. Komesu, H.-K. Jeong, and P. A. Dowben, Europhys. Lett. 43, 624 (2000).

${ }^{11}$ T. Komesu, C. N. Borca, H.-K. Jeong, P. A. Dowben, D. Ristoiu, J. P. Nozières, Sh. Stadler, and Y. U. Idzerda, Phys. Lett. A 273, 245 (2000).

${ }^{12} \mathrm{P}$. Y. Yu and M. Cardona, Fundamentals of Semiconductors (Springer, Berlin, 1996).

${ }^{13}$ F. García-Moliner and F. Flores, Introduction to the Theory of Solid Surfaces (University Press, Cambridge, 1979).

${ }^{14}$ W. Jones and N. H. March, Theoretical Solid State Physics I (Wiley, London, 1973).

${ }^{15}$ A. Miller and P. A. Dowben, J. Phys.: Condens. Matter 5, 5459 (1993).

${ }^{16}$ R. Skomski, C. Waldfried, and P. A. Dowben, J. Phys.: Condens. Matter 10, $5833(1998)$. 